\title{
Gut microbiome, obesity, and metabolic dysfunction
}

\author{
Herbert Tilg ${ }^{1}$ and Arthur Kaser ${ }^{2}$ \\ ${ }^{1}$ Christian Doppler Research Laboratory for Gut Inflammation, Medical University Innsbruck, Innsbruck, Austria. \\ 2Division of Gastroenterology and Hepatology, Department of Medicine, University of Cambridge, Cambridge, United Kingdom.
}

\begin{abstract}
The prevalence of obesity and related disorders such as metabolic syndrome has vastly increased throughout the world. Recent insights have generated an entirely new perspective suggesting that our microbiota might be involved in the development of these disorders. Studies have demonstrated that obesity and metabolic syndrome may be associated with profound microbiotal changes, and the induction of a metabolic syndrome phenotype through fecal transplants corroborates the important role of the microbiota in this disease. Dietary composition and caloric intake appear to swiftly regulate intestinal microbial composition and function. As most findings in this field of research are based on mouse studies, the relevance to human biology requires further investigation.
\end{abstract}

\section{Introduction}

The prevalence of obesity and the associated disorders metabolic syndrome and type 2 diabetes (T2D) have increased substantially worldwide over the last decades. Obesity increases risk for many other diseases such as atherosclerosis, nonalcoholic fatty liver disease, and certain cancers. Recent insight suggests that the intestinal microbial flora could play an important role in obesity and its related diseases.

The human intestine harbors an enormously complex, diverse, and vast microbial community, referred to as gut microflora or microbiota (1-4). The human gut microbiota is estimated to consist of at least $10^{14}$ bacteria and archaea, composed of approximately 1,100 prevalent species, with approximately 160 such species per individual. In its entirety, the microflora is estimated to contain 150-fold more genes than our own host genomes (5). Apart from contributing substantial beneficial functions to the host (e.g., digestion of otherwise indigestible plant polysaccharides), this separate ecosystem has enormous potential for physiological and pathological interactions with the host; for example, we have already learned that the microbiota drives the development of the mucosal and systemic immune system and controls the regeneration of the intestinal epithelium $(6,7)$.

The development of obesity and the metabolic syndrome is a complex process involving genetic and environmental factors and is associated with pathways that connect metabolism with the immune system and vice versa (8-15). Important studies on the relationship of the intestinal microbial flora with obesity have uncovered profound changes in the composition and metabolic function of the gut microbiota in obese individuals (16-19), which appear to enable the "obese microbiota" to extract more energy from the diet (20). Moreover, these studies have demonstrated that the gut microbiota interacts with host epithelial cells to indirectly control energy expenditure and storage (16).

Genetic investigations have identified multiple genes that confer increased risk for obesity that individually may have comparatively modest effects on hunger, satiety, and food intake (21-23). However, these minor effects may be amplified in the current envi-

Conflict of interest: The authors have declared that no conflict of interest exists. Citation for this article: J Clin Invest. 2011;121(6):2126-2132. doi:10.1172/JCI58109. ronment, in which, in many parts of the world, nearly unlimited amounts of food have been available during the last decades. In this article we will discuss current evidence on how the intestinal microbiota might have a profound role in the development of obesity and the metabolic syndrome and thereby could contribute to the obesity epidemic.

\section{Gut microbiota in animal and human obesity: evidence of disturbance}

New molecular, culture-independent techniques that are based on microbial DNA sequencing have profoundly transformed our ability to study microbial communities $(24,25)$. These techniques have demonstrated that the mammalian gut microbiota belongs predominantly to four bacterial phyla: the Gram-negative Bacteroidetes and Proteobacteria and the Gram-positive Actinobacteria and Firmicutes. Initial evidence for an altered microflora associated with obesity came from studies in the leptin-deficient $o b / o b$ mouse model. 16S rRNA sequencing of the distal gut microbiota of $o b / o b$ mice, lean $o b /+$, and wild-type siblings and their $o b /+$ mothers, all fed the same diet, revealed that $o b / o b$ mice exhibit a major reduction in the abundance of Bacteroidetes and a proportional increase in Firmicutes (17). Feeding of a high-fat/highpolysaccharide diet to genetically wild-type rodents led to similar microbial changes (26). Confounding factors affecting microbial composition and function may include diet per se (discussed below), the use of antibiotics (and other drugs), which substantially reduce bacterial diversity (27), and possibly effects related to the genetic background of animal models (28).

Consistent with animal models, Ley et al. observed analogous differences with an increase in the ratio of Firmicutes/Bacteroidetes in the distal gut microbiota in human obesity (29). Another study demonstrated that Firmicutes were dominant in lean and obese individuals and decreased in 3 patients undergoing Rouxen-Y gastric bypass surgery (30). In contrast to earlier studies, Zhang et al. (30) described that Prevotellaceae, a subgroup of Bacteroidetes, are significantly enriched in obesity, again raising the potentially important issue of diet as a confounding factor, as the patients in the Ley study (29) were either on a fat-restricted or carbohydrate-restricted diet, whereas in the Zhang study, researchers did not limit dietary components. Another study also described a decrease of Bacteroidetes in obesity and an increase in 


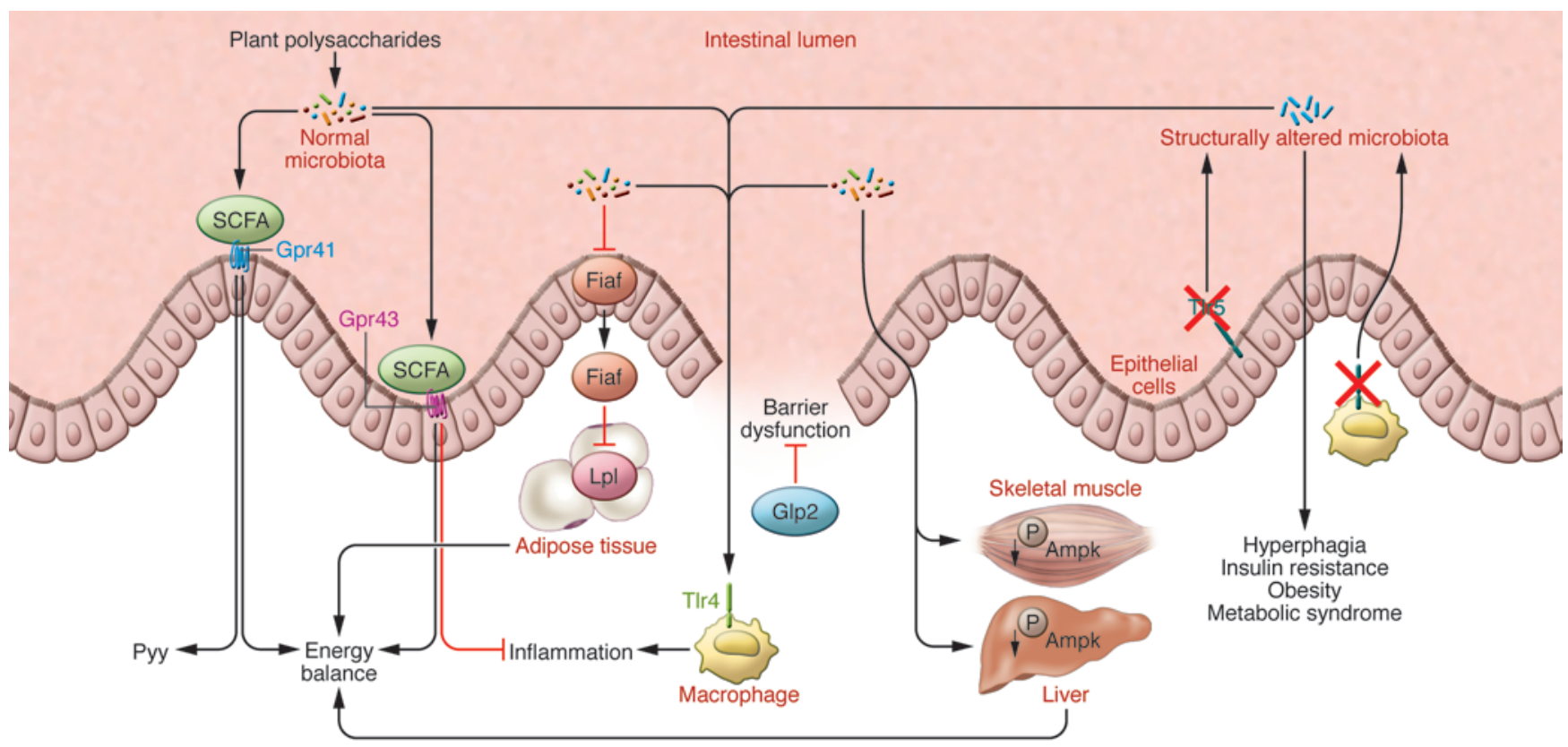

Figure 1

Microbiota regulates host metabolic functions. The microbiota controls host physiology at multiple levels. Microbial metabolic products such as SCFAs bind to GPCRs on intestinal epithelial cells (for example, Gpr41 and Gpr43) to control energy balance, partly via the gut-derived hormone Pyy, and also to control the inflammatory responsiveness of the host. TIr5 activation (e.g., through bacterial flagellin) presumably on epithelial or myeloid cells profoundly affects the structural composition of the intestinal microbiota, which in turn regulates appetite, weight gain, and insulin sensitivity through unknown mechanisms. Microbial signals also regulate Fiaf release from intestinal epithelial cells, which acts as an inhibitor of $\mathrm{Lpl}$ and thereby regulates peripheral fat storage. Through another unknown mechanism, the microbiota also regulates the energy gauge in the liver and muscle through the phosphorylation of Ampk. Glp2 ascertains epithelial barrier function, and a leaky barrier leads to exposure and activation of myeloid cells in response to microbial signals such as the Tlr4 ligand endotoxin. Fiaf, fasting-induced adipose factor; Glp2, glucagonlike peptide-2; Gpr41/43, G-protein coupled receptor; Lpl, lipoprotein lipase; Pyy, peptide YY; SCFA, short chain fatty acids.

Firmicutes (e.g., Lactobacilli) (31). Overweight pregnant patients (week 24) also have reduced numbers of Bifidobacteria and Bacteroidetes, whereas increased numbers of certain Firmicutes (e.g., Staphylococcus) or Proteobacteria (e.g., Enterobacteriaceae such as Escherichia coli) were detected (32).

However, it is worthwhile to note that the aforementioned "typical" changes in the gut microbiota in human obesity have not been found by all investigators $(33,34)$. Schwiertz and colleagues reported even lower ratios of Firmicutes to Bacteroidetes in obese human adults compared with lean controls (33). Another study also observed no differences between obese and non-obese subjects in the number of Bacteroidetes measured in fecal samples, and no significant changes using weight loss diets. However, significant dietdependent reductions in a group of butyrate-producing Firmicutes were found (34). A fascinating paper has been recently presented by Arumugam et al. (35) investigating the phylogenetic composition of 39 fecal samples from individuals representing 6 nationalities. Combining those data with previously published data sets, the authors characterized three clusters of individual microbiotal composition referred to as enterotypes that were not nation- or continent-specific. Interestingly, their results did not reveal any correlation between body mass index and the Firmicutes/Bacteroidetes ratio. Their analysis, however, suggested that metagenomic-derived functional biomarkers might be more important than phylogenetic ones, as they identified three marker molecules that correlate strongly with the host's body mass index, two of which are ATPase complexes, supporting the link found between energy harvest and obesity in the host (20). Varying data in several human studies further stress the importance of potential confounding factors such as diet or day-to-day variability in microbiotal makeup, and the need for well-controlled study designs. Whereas a few studies have observed an increase in the Firmicutes/Bacteroidetes ratio (29, 31, 32), others have failed to demonstrate such a correlation (30, 33-35). Therefore, differences at the phylum level are probably less important than metagenomic-based functional aspects.

\section{Energy harvest affected by the microbiota}

The gut microbiota benefits the host in numerous ways, among them contributing the capability to extract calories from otherwise indigestible common polysaccharides in the diet (36) via enzymes such as glycoside hydrolases and others that are not encoded within the human genome $(37,38)$. Studies in germ-free mice revealed that the gut microbiota enhances adiposity mainly by increased energy extraction from food and by regulating fat storage $(16,39)$, and germ-free mice are protected from obesity and metabolic syndrome $(16,17,40)$. Specifically, conventionalization (the restoration of conventional intestinal flora) of germ-free mice resulted in a substantial increase in body fat, hepatic triglycerides, fasting plasma glucose, and insulin resistance. The presence of a microbial population enhanced intestinal monosaccharide uptake, resulting in increased de novo lipogenesis and accumulation of hepatic and adipose tissue triglycerides. Apart from these general 
effects of the microbiota on food utilization, $o b / o b$ mice have been reported to harvest energy from food more efficiently than lean wild-type animals (41). A metagenomic sequencing analysis and metabolic pathway reconstruction of the distal intestinal flora of $o b / o b$ mice revealed that the changes in the relative abundance of Bacteroidetes and Firmicutes were associated with a switch in the metabolic potential of the microbiota that conferred the $o b / o b$ gut microbiome an increased capacity to harvest energy from the diet (20). Remarkably, this trait of obesity was transmissible through fecal transplants from obese (as compared to non-obese) to germfree mice $(20,26)$. Ob/ob mice also harbor more methanogenic archaea, which may increase efficiency of bacterial fermentation via removal of $\mathrm{H}_{2}(20)$. Studies with Methanobrevibacter smithii, and Bacteroides thetaiotaomicron revealed that co-colonization not only enhanced efficiency, but also changed the specificity of bacterial polysaccharide fermentation, increasing adiposity compared with mice colonized with either organism alone (42).

Further studies have revealed additional complexities related to the potential increased energy harvest from the diet through dietary-induced or genetically induced obese microbiota (43). In this study, the authors showed that both age and diet are important factors not only for the composition of the gut microbiota but also for its potential to extract energy. Experimental protocols that allow colonization of germ-free mice with selective human flora are essential to investigate the effects of various diets and other confounding factors such as age on the microbiota and their consequent implications for host metabolism (44).

\section{Effects of diet and other factors on the composition of the gut microbiome}

Several lines of evidence suggest that dietary factors might profoundly influence microbiotal composition. Studies in resistin-like molecule $\beta$-knockout mice, which are resistant to diet-induced obesity, revealed that dietary factors are the key determinant of microbial composition, and indeed appear more relevant than obesity per se (45). In this study, switching to a high-fat diet resulted in a decrease in Bacteroidetes, whereas the numbers of Firmicutes and Proteobacteria increased. Importantly, this was observed in both the presence and absence of obesity, clearly suggesting that diet must be considered as a confounding factor affecting microbial composition. In another study, fresh or frozen adult human fecal microbial communities from lean donors were transplanted into germ-free C57BL/6J mice (44). These microbially humanized mice established a stable and heritable microbiota that reproduced much of the bacterial diversity of the donor's microbiota. A change in the diet (i.e., from a low-fat, plant polysaccharide to a high-fat, high-sugar diet) shifted the structure of the microbiota within a single day, along with changes in metabolic pathways in the microbiome. When fed a Western diet, these microbially humanized mice exhibited increased adiposity, and this trait was transferable via microbiota transplantation (44). The genetic background of our microbiota might determine how certain dietary factors are handled. Genetic and functional differences between Bacteroides spp. are predictive how these bacteria utilize fructans, a class of fructose-based polysaccharides (46). Undoubtedly, diet critically affects the gut microbiome, changes occur very rapidly, and adiposity might be transferable by fecal transplantation.

The intestinal microbiota from children ingesting a modern Western diet and a rural African diet may differ on the same basis (47). Children from Burkina Faso showed a significant enrich- ment in Bacteroidetes and a depletion of Firmicutes, with a significant abundance of bacteria from the genus Prevotella and Xylanibacter, which are known to encode genes enabling hydrolysis of cellulose and xylan. These African children indeed demonstrated a higher content of short-chain fatty acids (SCFAs). Gut microbiota in rural Africa may allow individuals to maximize energy intake from fibers while protecting from inflammation and infection (47). Genetic and environmental factors may have also contributed significantly to the dramatic differences in gut microbiome composition observed in these children.

Besides diet, genetic and other environmental factors may shape the gut's microbiota. Benson et al. found in mice a core measurable microbiota of 64 taxonomic groups that varied across most animals, largely dependent on individual host genotype (48). This study identified host quantitative trait loci able to control individual microbial species. The complexity of factors affecting the gut microbiome early in life has been convincingly demonstrated in a recent study by Koenig et al. (49). Most "chaotic shifts" in the microbiome were associated with exceptional life events, and interestingly, species harboring functional genes involved in the fermentation of plant polysaccharides were even present before the introduction of solid foods. Overall, phylogenetic diversity seems to develop gradually over time. These studies are in accordance with the recent finding that obese and lean twins share a core microbiome at the gene rather than at the phylum level (40).

\section{Obesity, microbiota, and epithelial integrity}

Some lines of experimental evidence suggest that high-fat diets may affect epithelial integrity and hence lead to impaired gut permeability, and consequently to systemic inflammation via translocation of Tlr ligands (50). Prebiotic carbohydrates and/or antibiotics lowered systemic endotoxin levels and inflammatory cytokine expression in the liver (50). Such improvement of metabolic inflammation in obese mice might not only involve changes in the microbiota, but also expression of glucagon-like peptide 2 (Glp2) (51), an intestinal growth factor with anti-inflammatory activities (52) that stabilizes intestinal barrier function (53). Prebiotic therapy improved intestinal permeability, systemic inflammation, hepatic expression of pro-inflammatory cytokines, and insulin sensitivity in $o b / o b$ mice, which was paralleled by enhanced intestinal Glp2 expression. Treatment of animals with a Glp2 agonist revealed similar beneficial effects (51). The endocannabinoid system has also been shown to potentially mediate the influence of microbiota on gut permeability. Studies involving specific antagonists and agonists demonstrated that the endocannabinoid system controls not only gut permeability but also plasma LPS levels and adipogenesis (54). Endocannabinoids increase mRNA expression of the tight junction protein occludin-1 and decrease expression of claudin-1, further supporting a potential role in the regulation of intestinal permeability (55).

Sprague-Dawley rats typically present either with an obesityprone or an obesity-resistant phenotype. This model therefore could be an attractive way to dissect diet from associated obesity/ inflammation. When fed a high-fat diet, only the obesity-prone rats show an increase in ileal Tlr4 expression associated with ileal inflammation (56). Furthermore, intestinal permeability and serum endotoxin levels were increased in obesity-prone, but not obesity-resistant rats. However, the diet induced identical microbiota changes in both groups. Hence, although a high-fat diet may affect the microbiota, other (host) factors might determine intes- 
tinal barrier function and induction of local/systemic inflammation. Indeed, multiple interactions between certain dietary factors, the microbiota, or their products and the innate immune system may take place and affect barrier function. For example, dietary wheat gluten proteins (gliadin) decrease the number of goblet cells in the small intestine, especially in conjunction with certain intestinal bacteria (e.g., Escherichia coli), and lead to impairment of tight junctions (57). Antibiotic therapy may be another factor affecting epithelial integrity, as treatment with metronidazole reduces mucus thickness and thereby predisposes mice to exacerbated Citrobacter rodentium-induced colitis (58). Furthermore, widespread use of antibiotics in early life has been suggested as a link to the obesity epidemic (4).

However, it remains to be unambiguously determined whether gastrointestinal barrier function is indeed impaired in human obesity. In fact, a recent study on 13 obese and 11 control subjects did not find evidence of impaired barrier function despite systemically elevated levels of C-reactive protein, a measure of inflammation (59). Further studies are needed to better define epithelial integrity in human obesity and its potential role in the microbiota.

\section{Gut microbiota regulate host genes that control metabolic processes}

Colonization of germ-free mice substantially alters transcription of various mediators in the intestine (particularly in epithelial cells), thereby regulating key intestinal functions such as nutrient absorption, mucosal barrier function, metabolic functions, and angiogenesis (60). Fasting-induced adipose factor (Fiaf; also known as angiopoietin-like protein 4) is a circulating lipoprotein lipase (Lpl) inhibitor produced by the intestine, liver, and adipose tissue (61). Conventionalization of germ-free mice suppresses expression of Fiaf in gut epithelial cells (16). Increased adipocyte $\mathrm{Lpl}$ activity results in increased cellular uptake of fatty acids and adipocyte triglyceride accumulation. Germ-free Fiaf ${ }^{\prime-}$ mice contain the same amount of total body fat weight as conventionalized (i.e., Fiaf-suppressed) mice, suggesting that Fiaf is a mediator of microbial regulation of energy storage (16). In contrast, mice fed a high-fat diet complemented with Lactobacillus paracasei exhibited significantly reduced body fat, which was paralleled by increased circulating levels of Fiaf (62). Lactobacillus paracasei indeed upregulated Fiaf expression in colonic epithelial cell lines, and oral inoculation of germ-free mice with this species resulted in increased circulating Fiaf levels (62). Hypothalamic Fiaf expression is regulated by physiological appetite regulators and mediates their anorexigenic effects via inhibition of hypothalamic AMPK activity. Therefore, Fiaf appears to have an important role in central regulation of energy metabolism (63). Manipulation of Fiaf by the microflora seems to be a prototypic model of its remote control of host physiology (Figure 1).

Another pathway that has been suggested to account for the consistently lean phenotype of germ-free mice on a high-fat diet involves Ampk (39). Ampk is a key enzyme conserved from yeast to humans and acts as a fuel gauge that controls cellular energy status (64). The lean phenotype of germ-free mice on a Western diet is associated with increased levels of phosphorylated Ampk in skeletal muscle and liver, increased levels of its downstream targets involved in fatty acid oxidation, such as acetyl-CoA carboxylase or carnitine-palmitoyltransferase, decreased glycogen storage, and increased hepatic insulin sensitivity (39). Therefore, our microbiota might affect skeletal muscle fatty acid oxidation through metabolic pathways involving Ampk (Figure 1). The exact pathway whereby the microbiota signals to liver or skeletal muscle Ampk is unclear but appears to be independent from Fiaf (39).

A third pathway that influences host energy storage also involves intestinal epithelial cells as sensors of microbial products. The gut microbiota synthesizes a broad spectrum of hydrolases (65) that digest complex dietary carbohydrates to monosaccharides and SCFAs such as acetate, propionate, and butyrate. As end products of bacterial fermentation, these SCFAs represent an important energy source. SCFAs not only diffuse passively into the circulation, but may also act in the gut as signaling molecules. Propionate and acetate are ligands for two G protein-coupled receptors (GPCRs), Gpr41 and Gpr43, mainly expressed by intestinal epithelial cells $(66,67)$. Conventionally raised $\mathrm{Gpr} 41^{-/-}$mice and germ-free Gpr41-/mice colonized with only Bacteroides thetaiotaomicron and Methanobrevibacter smithii are significantly leaner than wild-type littermates despite similar levels of chow consumption, while there are no differences between wild-type or Gpr41//- germ-free mice (68). These studies showed that Gpr41 might be a regulator of host energy balance through effects that are dependent upon the gut microbiota and their metabolic capacity (ref. 68 and Figure 1). Upon activation of GPCRs, propionate and acetate induce the release of peptide YY (Pyy). Pyy, an enteroendocrine cell-derived hormone that normally inhibits gut motility and accelerates intestinal transit rate, might be

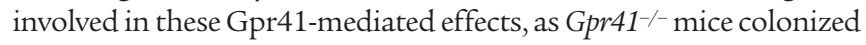
with only Bacteroides thetaiotaomicron and Methanobrevibacter smithii demonstrated increased circulating Pyy levels, an increased transit time, and reduced calorie extraction from the diet (68). Gpr41 also mediates SCFA-induced synthesis of leptin, an adipocytokine with pleiotropic effects on appetite and energy metabolism (69).

SCFAs also act as ligands of Gpr43, and Gpr43-/- mice appear protected from high-fat diet-induced obesity and insulin resistance, at least partly due to Gpr43-regulated energy expenditure (70). Moreover, stimulation of Gpr43 by SCFAs limits inflammation in experimental models of colitis, arthritis, and asthma (71). Germ-free mice, devoid of SCFAs due to the absence of bacteria that would ferment dietary fiber, exhibited exacerbated inflammation in these models, similar to Gpr43-/- mice (71). Gpr43 might provide a molecular link between diet, gastrointestinal bacterial metabolism, and immune and inflammatory responses (71) and could possibly also play some role in colon carcinogenesis (72).

In summary, the gut microbiota affects host energy expenditure and metabolic and immune/inflammatory functions via several pathways. The intestinal epithelium is at the interface between environment, microbiota, and host and plays a substantial and remarkable role in all of these processes.

\section{Microbiota and metabolic syndrome}

Metabolic syndrome is thought to develop through the interaction of various genetic and environmental factors. A complex and still poorly characterized interaction between the intestinal microbiota and the innate immune system may be involved in metabolic dysfunction (73). Metabolic syndrome, diabetes, and obesity are characterized by low-grade inflammation, and adipokines play a central role (74). Ob/ob mice treated with antibiotics (norfloxacin and ampicillin) exhibit changes in the microbiota and an improvement in insulin resistance, fasting glycemia, and glucose tolerance compared with control $o b / o b$ mice (75), along with a reduction in systemic "metabolic" endotoxemia and inflammatory parameters (50). In a study involving genetic (apoA-I-deleted) and diet- 
induced murine models of metabolic syndrome, in addition to profound overall changes in glucose tolerance, barrier-protective bacterial species (Bifidobacterium spp.) were reduced and endotoxin-producing species (Desulfovibrionaceae) were increased (76). Diet appeared to be a substantially stronger contributor to structural microbial changes compared with the genetic alteration in this study (76). In addition to regulating insulin sensitivity, the presence or absence of a microbial flora might also regulate cholesterol metabolism (77). Some evidence indicates that T2D, irrespective of obesity, might also affect the structural composition of the microbiota, as might be expected, with differences noted between obese patients and type 2 diabetics $(78,79)$. T2D might be associated with the dominance of gram-negative bacteria in the gut, such as Bacteroidetes (80), and in fact a decrease in Bacteroides-Prevotella spp. has been associated with improved metabolic endotoxemia and decreased systemic inflammatory markers in diabetic mice. Specific prebiotics such as oligofructose might affect the structural composition of the microbiota upon high-fat diet feeding, which might improve parameters of metabolic inflammation (80).

A recent report revealed a profound effect of the innate immune receptor, the pattern recognition receptor Tlr5, on structural microbial composition and the consequences for the pathogenesis of metabolic syndrome (81). Tlr5 $5^{-/-}$mice exhibit hyperphagia and develop a metabolic syndrome characterized by hyperlipidemia, hypertension, insulin resistance, and obesity (81). Food restriction prevented many of the metabolic abnormalities observed in Tlr $5^{-/-}$mice, but even lean $T l r 5^{-/-}$mice exhibited insulin resistance. Notably, metabolic changes in $\mathrm{Tlr}^{-/-}$mice resulted in alterations in the composition of the gut microbiota, and transfer of the altered microbiota from $\mathrm{Tlr}^{-/-}$mice into germ-free wild-type mice conferred a metabolic syndrome phenotype to the latter (ref. 81 and Figure 1). These data not only provide experimental evidence that innate immune signaling is critical in the induction of metabolic syndrome, but notably show that alterations in our intestinal microbiota can be sufficient to induce the metabolic syndrome. This report, however, has been challenged recently by a study in two different animal colonies with Tlr5 deficiency, where intestinal inflammatory disease or metabolic dysfunction was not evident (82). These authors only observed an impaired CD4 T cell response to flagellated pathogens. One might speculate that differences in the gut microbiota between institutional animal facilities or differences obtained during rederivation of these animals might explain divergent phenotypes in Tlr5-deficient mice.

Transfer of disease phenotypes via the microbiota has also been reported for colitis (83) and obesity (20). The fascinating triangular relationship between the microbiota, the innate immune system, and metabolic function allows for a completely new perspective on related diseases, which raises many important questions: What are the roles of other TLRs beyond TLR5? Which intestinal disturbances (e.g., infections) may have long-lasting effects on metabolic diseases? Which microbiota-derived metabolic products beyond SCFAs interact with the innate immune system?

Besides immune and inflammatory mechanisms, other pathways may be involved in the link between gut microbiota and metabolic syndrome. Our microbiota produces enzymes that degrade ingested polysaccharides, thereby promoting the absorption of nutrients (especially carbohydrates), resulting in increased liver lipogenesis, hepatic insulin resistance, and hyperinsulinemia. It has been demonstrated that high intake of cereal fiber is associated with reduced risk for T2D. Various dietary components including wheat fiber, inulin, oat $\beta$-glucan, or starch with high amylase content affect glucose absorption, decrease insulin secretion, increase concentrations of the incretin Glp1, and increase SCFA production and absorption (84-86). None of these studies, however, have assessed the effects of diet on the gut microbiota (84-86).

\section{Unanswered questions, future directions, and concluding remarks}

The mammalian gut microbiota has been studied for decades, and the advent of new technologies during the last few years has allowed for a grand leap forward and has generated important novel insights. However, we are nonetheless still scratching at the surface of what - until recently - had to be considered a "black box". While large-scale sequencing of $16 \mathrm{~S}$ ribosomal DNA genes has revealed the enormous interindividual variation and inherent complexity of the microbiota, more recent metagenomic approaches now allow us to estimate that the microbial gene content is 150fold larger than the human genome, and most of these genes are of unknown function. Needless to say, we can barely imagine the many functional implications of this enormous gene pool for the mutual relationship of the microbiome with the host. In addition, we still know very little about other constituents of the microbial world of the intestine, such as viruses and fungi that might - similar to bacterial components - affect host metabolism, immunity, and physiology in general.

A key aspect will be to gain a proper understanding of environmental influences on the microbiota and what the consequences are of such structural and functional changes within the microbiota on metabo-inflammatory diseases. These studies will extend well beyond the initial studies on diet discussed herein; for example, it will be important to reveal potential long-term consequences of antibiotic therapies at various ages of life. In that context, does a "disappearing microbiota", as recently proposed by Blaser and Falkow (4), play a role in obesity and its consequences? It is indeed striking that a common theme associated with diseases or conditions as diverse as obesity and inflammatory bowel disease is the substantially reduced microbial complexity as compared with the intestinal microbial communities of healthy subjects $(17,20,29,87)$. The recently reported strategy for "humanizing" the murine microbiota (44) might offer great potential to study environmental influences on the microbiome in the context of specific host genotypes.

It is fascinating to speculate that the gut microbiome might contain a critical intestinal trigger linking environment and host in obesity. Fecal transplants have revealed promising results in the treatment of Clostridium difficile infection (88) and could become another interesting option for the therapy of obesity and metabolic syndrome. Indeed, a first small human study on fecal transplantation in patients with metabolic syndrome, though as yet only presented in abstract form, hinted toward improved insulin sensitivity (89). It has to be stated that such an approach might encounter many pitfalls and challenges, such as the complexity of dietary factors, selection and preparation of donors, timing of intervention, current medication, and antibiotic pretreatment. Apart from the likely reservations of patients and physicians, there are still many important questions that must be answered before this can be tested in a broader fashion.

Various host pathways, mainly emanating from epithelial cells, have been characterized in the last years that might mediate the effects of microbiota on metabolism. These factors include Fiaf, 
Ampk, Gpr41, Gpr43, Glp2, the endocannabinoid system, and more. The interaction of the intestinal microbial world with its host, and its mutual regulation, will become one of the important topics of biomedical research and will provide us with further insights at the interface of microbiota, metabolism, metabolic syndrome, and obesity. A better understanding of the interaction between certain diets and our human gut microbiome should help to develop new guidelines for feeding humans at various time points in their life, help to improve global human health, and establish ways to prevent or treat various food-related diseases. Finally, we must be aware that most of the data discussed herein, though fascinating, are based on murine studies commonly using knockout or germ-free animals. Their relevance in human biology will require much more research.

\section{Acknowledgments}

Work in the authors' laboratories is supported by the Christian Doppler Research Society (grant to H. Tilg), the European Research Council (grant 260961; to A. Kaser), and the National Institute for Health Research Cambridge Biomedical Research Centre. The Obesity Review Series is supported in part by unrestricted educational grants from Merck \& Co. and the Life Sciences Institute of the University of Michigan.

Address correspondence to: Herbert Tilg, Christian Doppler Research Laboratory for Gut Inflammation, Medical University Innsbruck, Anichstrasse 35, 6020 Innsbruck, Austria. Phone: 43.512.504.23374; Fax: 43.512.504.6723374; E-mail: herbert.tilg@i-med.ac.at.
1. Hooper LV, Gordon JI. Commensal host-bacterial relationships in the gut. Science. 2001; 292(5519):1115-1118.

2. Ley RE, Peterson DA, Gordon JI. Ecological and evolutionary forces shaping microbial diversity in the human intestine. Cell. 2006;124(4):837-848.

3. Neish AS. Microbes in gastrointestinal health and disease. Gastroenterology. 2009;136(1):65-80.

4. Blaser MJ, Falkow S. What are the consequences of the disappearing human microbiota? Nat Rev Microbiol. 2009;7(12):887-894

5. Qin J, et al. A human gut microbial gene catalogue established by metagenomic sequencing. Nature. 2010;464(7285):59-65.

6. Slack E, et al. Innate and adaptive immunity cooperate flexibly to maintain host-microbiota mutualism. Science. 2009;325(5940):617-620.

7. Lee YK, Mazmanian SK. Has the microbiota played a critical role in the evolution of the adaptive immune system? Science. 2010;330(6012):1768-1773.

8. Frayling TM, et al. A common variant in the FTO gene is associated with body mass index and predisposes to childhood and adult obesity. Science. 2007;316(5826):889-894.

9. Cecil JE, Tavendale R, Watt P, Hetherington MM, Palmer CN. An obesity-associated FTO gene variant and increased energy intake in children. NEngl JMed. 2008;359(24):2558-2566.

10. Loos RJ, et al. Common variants near MC4R are associated with fat mass, weight and risk of obesity. Nat Genet. 2008;40(6):768-775.

11. Chambers JC, et al. Common genetic variation near MC4R is associated with waist circumference and insulin resistance. Nat Genet. 2008;40(6):716-718.

12. Thorleifsson G, et al. Genome-wide association yields new sequence variants at seven loci that associate with measures of obesity. Nat Genet. 2009; 41(1):18-24.

13. Willer CJ, et al. Six new loci associated with body mass index highlight a neuronal influence on body weight regulation. Nat Genet. 2009;41(1):25-34.

14. Sabatti C, et al. Genome-wide association analysis of metabolic traits in a birth cohort from a founder population. Nat Genet. 2009;41(1):35-46.

15. Meyre D, et al. Genome-wide association study for early-onset and morbid adult obesity identifies three new risk loci in European populations. Nat Genet. 2009;41(2):157-159.

16. Backhed F, et al. The gut microbiota as an environmental factor that regulates fat storage. Proc Natl Acad SciU S A. 2004;101(44):15718-15723.

17. Ley RE, Backhed F, Turnbaugh P, Lozupone CA, Knight RD, Gordon JI. Obesity alters gut microbial ecology. Proc Natl Acad Sci U S A. 2005; 102(31):11070-11075.

18. Turnbaugh PJ, Ley RE, Hamady M, Fraser-Liggett CM, Knight R, Gordon JI. The human microbiome project. Nature. 2007;449(7164):804-810.

19. Turnbaugh PJ, Gordon JI. The core gut micro- biome, energy balance and obesity. J Physiol. 2009;587(pt 17):4153-4158.

20. Turnbaugh PJ, Ley RE, Mahowald MA, Magrini V, Mardis ER, Gordon JI. An obesity-associated gut microbiome with increased capacity for energy harvest. Nature. 2006;444(7122):1027-1031.

21. O'Rahilly S. Human genetics illuminates the paths to metabolic disease. Nature. 2009;462(7271):307-314.

22. O'Rahilly S, Farooqi IS. Human obesity as a heritable disorder of the central control of energy balance. Int J Obes (Lond). 2008;32 suppl 7:S55-S61.

23. McCarthy MI. Genomics, type 2 diabetes, and obesity. N Engl J Med. 2010;363(24):2339-2350.

24. Eckburg PB, et al. Diversity of the human intestinal microbial flora. Science. 2005;308(5728):1635-1638.

25. Andersson AF, Lindberg M, Jakobsson H, Backhed F, Nyren P, Engstrand L. Comparative analysis of human gut microbiota by barcoded pyrosequencing. PLoS One. 2008;3(7):e2836.

26. Turnbaugh PJ, Backhed F, Fulton L, Gordon JI. Diet-induced obesity is linked to marked but reversible alterations in the mouse distal gut microbiome. Cell Host Microbe. 2008;3(4):213-223.

27. Dethlefsen L, Huse S, Sogin ML, Relman DA. The pervasive effects of an antibiotic on the human gut microbiota, as revealed by deep $16 \mathrm{~S}$ rRNA sequencing. PLoS Biol. 2008;6(11):e280.

28. Fleissner CK, Huebel N, Abd El-Bary MM, Loh G, Klaus S, Blaut M. Absence of intestinal microbiota does not protect mice from diet-induced obesity. BrJ Nutr. 2010;104(6):919-929.

29. Ley RE, Turnbaugh PJ, Klein S, Gordon JI. Microbial ecology: human gut microbes associated with obesity. Nature. 2006;444(7122):1022-1023.

30. Zhang $\mathrm{H}$, et al. Human gut microbiota in obesity and after gastric bypass. Proc Natl Acad Sci U S A. 2009;106(7):2365-2370.

31. Armougom F, Henry M, Vialettes B, Raccah D, Raoult D. Monitoring bacterial community of human gut microbiota reveals an increase in Lactobacillus in obese patients and Methanogens in anorexic patients. PLoS One. 2009;4(9):e7125.

32. Santacruz A, et al. Gut microbiota composition is associated with body weight, weight gain and biochemical parameters in pregnant women. BrJ Nutr. 2010;104(1):83-92.

33. Schwiertz A, et al. Microbiota and SCFA in lean and overweight healthy subjects. Obesity (Silver Spring). 2010;18(1):190-195.

34. Duncan SH, et al. Human colonic microbiota associated with diet, obesity and weight loss. Int J Obes (Lond). 2008;32(11):1720-1724.

35. Arumugam M, et al. Enterotypes of the human gut microbiome [published online ahead of print April 20, 2011]. Nature. doi:10.1038/nature09944.

36. Flint HJ, Bayer EA, Rincon MT, Lamed R, White BA. Polysaccharide utilization by gut bacteria: potential for new insights from genomic analysis. Nat Rev Microbiol. 2008;6(2):121-131.
37. Xu J, et al. A genomic view of the human-Bacteroides thetaiotaomicron symbiosis. Science. 2003;299(5615):2074-2076.

38. Sonnenburg JL, et al. Glycan foraging in vivo by an intestine-adapted bacterial symbiont. Science. 2005;307(5717): 1955-1959.

39. Backhed F, Manchester JK, Semenkovich CF, Gordon JI. Mechanisms underlying the resistance to diet-induced obesity in germ-free mice. Proc Natl Acad Sci U S A. 2007;104(3):979-984.

40. Turnbaugh PJ, et al. A core gut microbiome in obese and lean twins. Nature. 2009;457(7228):480-484

41. Ferraris RP, Vinnakota RR. Intestinal nutrient transport in genetically obese mice. Am J Clin Nutr. 1995;62(3):540-546.

42. Samuel BS, Gordon JI. A humanized gnotobiotic mouse model of host-archaeal-bacterial mutualism. Proc Natl Acad Sci U S A. 2006;103(26):10011-10016.

43. Murphy EF, et al. Composition and energy harvesting capacity of the gut microbiota: relationship to diet, obesity and time in mouse models. Gut. 2010;59(12):1635-1642.

44. Turnbaugh PJ, Ridaura VK, Faith JJ, Rey FE, Knight $\mathrm{R}$, Gordon JI. The effect of diet on the human gut microbiome: a metagenomic analysis in humanized gnotobiotic mice. Sci Transl Med 1(6):6ra14.

45. Hildebrandt MA, et al. High fat diet determines the composition of the murine gut microbiome independently of obesity. Gastroenterology. 2009; 137(5):1716-1724.e1-e2.

46. Sonnenburg ED, et al. Specificity of polysaccharide use in intestinal bacteroides species determines diet-induced microbiota alterations. Cell. 2010;141(7):1241-1252.

47. De Filippo C, et al. Impact of diet in shaping gut microbiota revealed by a comparative study in children from Europe and rural Africa. Proc Natl Acad Sci U S A. 2010;107(33):14691-14696.

48. Benson AK, et al. Individuality in gut microbiota composition is a complex polygenic trait shaped by multiple environmental and host genetic factors. Proc Natl Acad Sci U S A. 2010;107(44):18933-18938.

49. Koenig JE, et al. Succession of microbial consortia in the developing infant gut microbiome. Proc Natl Acad Sci U S A. 2011;108 suppl 1:4578-4585.

50. Cani PD, et al. Changes in gut microbiota control metabolic endotoxemia-induced inflammation in high-fat diet-induced obesity and diabetes in mice. Diabetes. 2008;57(6):1470-1481.

51. Cani PD, et al. Changes in gut microbiota control inflammation in obese mice through a mechanism involving GLP-2-driven improvement of gut permeability. Gut. 2009;58(8):1091-1103.

52. Ivory CP, Wallace LE, McCafferty DM, Sigalet DL. Interleukin-10-independent anti-inflammatory actions of glucagon-like peptide 2. Am J Physiol Gastrointest Liver Physiol. 2008;295(6):G1202-G1210.

53. Benjamin MA, McKay DM, Yang PC, Cameron H, Perdue MH. Glucagon-like peptide-2 enhances 
intestinal epithelial barrier function of both transcellular and paracellular pathways in the mouse. Gut. 2000;47(1):112-119.

54. Muccioli GG, et al. The endocannabinoid system links gut microbiota to adipogenesis. Mol Syst Biol. 2010;6:392.

55. Alhamoruni A, Lee AC, Wright KL, Larvin M, O'Sullivan SE. Pharmacological effects of cannabinoids on the Caco- 2 cell culture model of intestinal permeability. J Pharmacol Exp Ther. 2010; 335(1):92-102

56. de La Serre CB, Ellis CL, Lee J, Hartman AL, Rutledge JC, Raybould HE. Propensity to high-fat diet-induced obesity in rats is associated with changes in the gut microbiota and gut inflammation. Am J Physiol Gas trointest Liver Physiol. 2010;299(2):G440-G448.

57. Cinova J, et al. Role of intestinal bacteria in gliadin-induced changes in intestinal mucosa: study in germ-free rats. PLoS One. 2011;6(1):e16169.

58. Wlodarska M, et al. Antibiotic treatment alters the colonic mucus layer and predisposes the host to exacerbated citrobacter rodentium-induced colitis. Infect Immun. 2011;79(4):1536-1545.

59. Brignardello J, Morales P, Diaz E, Romero J, Brunser O, Gotteland M. Pilot study: alterations of intestinal microbiota in obese humans are not associated with colonic inflammation or disturbances of barrier function. Aliment Pharmacol Ther. 2010;32(11-12):1307-1314

60. Hooper LV, Wong MH, Thelin A, Hansson L, Falk PG, Gordon JI. Molecular analysis of commensa host-microbial relationships in the intestine. Science. 2001;291(5505):881-884.

61. Yoon JC, et al. Peroxisome proliferator-activated receptor gamma target gene encoding a novel angiopoietin-related protein associated with adipose differentiation. Mol Cell Biol. 2000;20(14):5343-5349.

62. Aronsson L, et al. Decreased fat storage by Lactobacillus paracasei is associated with increased levels of angiopoietin-like 4 protein (ANGPTL4). PLoS One. 2010;5(9):e13087.

63. Kim HK, et al. Hypothalamic Angptl4/Fiaf is a novel regulator of food intake and body weight. Diabetes. 2010;59(11):2772-2780.

64. Li C, Keaney JF Jr. AMP-activated protein kinase: a stress-responsive kinase with implications for cardiovascular disease. Curr Opin Pharmacol.
2010;10(2):111-115.

65. Backhed F, Ley RE, Sonnenburg JL, Peterson DA, Gordon JI. Host-bacterial mutualism in the human intestine. Science. 2005;307(5717):1915-1920.

66 . Brown AJ, et al. The Orphan G protein-coupled receptors GPR41 and GPR43 are activated by propionate and other short chain carboxylic acids. J Biol Chem. 2003;278(13):11312-11319.

67. Le Poul E, et al. Functional characterization of human receptors for short chain fatty acids and their role in polymorphonuclear cell activation. J Biol Chem. 2003;278(28):25481-25489.

68 . Samuel BS, et al. Effects of the gut microbiota on host adiposity are modulated by the short-chain fatty-acid binding $\mathrm{G}$ protein-coupled receptor, Gpr41. Proc Natl Acad SciU S A. 2008;105(43):16767-16772

69. Zaibi MS, et al. Roles of GPR41 and GPR43 in leptin secretory responses of murine adipocytes to short chain fatty acids. FEBS Lett. 2010;584(11):2381-2386.

70. Bjursell $\mathrm{M}$, et al. Improved glucose control and reduced body fat mass in free fatty acid receptor 2-deficient mice fed a high-fat diet. Am J Physiol Endocrinol Metab. 2011;300(1):E211-E220.

71. Maslowski KM, et al. Regulation of inflammatory responses by gut microbiota and chemoattractant receptor GPR43. Nature. 2009;461(7268):1282-1286.

72. Tang Y, Chen Y, Jiang H, Robbins GT, Nie D. Gprotein-coupled receptor for short-chain fatty acids suppresses colon cancer. Int J Cancer. 2011; 128(4):847-856.

73. Cani PD, Delzenne NM. Interplay between obesity and associated metabolic disorders: new insights into the gut microbiota. Curr Opin Pharmacol. 2009;9(6):737-743.

74. Tilg H, Moschen AR. Adipocytokines: mediators linking adipose tissue, inflammation and immunity. Nat Rev Immunol. 2006;6(10):772-783.

75. Membrez M, et al. Gut microbiota modulation with norfloxacin and ampicillin enhances glucose tolerance in mice. FASEB J. 2008;22(7):2416-2426.

76. Zhang $\mathrm{C}$, et al. Interactions between gut microbiota, host genetics and diet relevant to development of metabolic syndromes in mice. ISME J. 2010;4(2):232-241.

77. Rabot S, et al. Germ-free C57BL/6J mice are resistant to high-fat-diet-induced insulin resistance and have altered cholesterol metabolism. FASEB J.
2010;24(12):4948-4959.

78. Wu X, et al. Molecular characterisation of the faecal microbiota in patients with type II diabetes. Curr Microbiol. 2010;61(1):69-78.

79. Larsen N, et al. Gut microbiota in human adults with type 2 diabetes differs from non-diabetic adults. PLoS One. 2010;5(2):e9085.

80. Cani PD, et al. Selective increases of bifidobacteria in gut microflora improve high-fat-diet-induced diabetes in mice through a mechanism associated with endotoxaemia. Diabetologia. 2007;50(11):2374-2383.

81. Vijay-Kumar M, et al. Metabolic syndrome and altered gut microbiota in mice lacking Toll-like receptor 5. Science. 2010;328(5975):228-231.

82. Letran SE, et al. TLR5-deficient mice lack basal inflammatory and metabolic defects but exhibit impaired CD4 $\mathrm{T}$ cell responses to a flagellated pathogen. J Immunol. 2011;186(9):5406-5412

83. Garrett WS, et al. Communicable ulcerative colitis induced by T-bet deficiency in the innate immune system. Cell. 2007;131(1):33-45

84. Freeland KR, Wilson C, Wolever TM. Adaptation of colonic fermentation and glucagon-like peptide-1 secretion with increased wheat fibre intake for 1 year in hyperinsulinaemic human subjects. $\mathrm{BrJ}$ Nutr. 2010;103(1):82-90.

85. Hooda S, Matte JJ, Vasanthan T, Zijlstra RT. Dietary oat beta-glucan reduces peak net glucose flux and insulin production and modulates plasma incretin in portal-vein catheterized grower pigs. J Nutr. 2010;140(9):1564-1569.

86. Regmi PR, van Kempen TA, Matte JJ, Zijlstra RT. Starch with high amylose and low in vitro digestibility increases short-chain fatty acid absorption, reduces peak insulin secretion, and modulates incretin secretion in pigs. J Nutr. 2011;141(3):398-405.

87. Frank DN, St Amand AL, Feldman RA, Boedeker EC, Harpaz N, Pace NR. Molecular-phylogenetic characterization of microbial community imbalances in human inflammatory bowel diseases. Proc Natl Acad Sci U S A. 2007;104(34):13780-13785.

88. Tvede M, Rask-Madsen J. Bacteriotherapy for chronic relapsing Clostridium difficile diarrhoea in six patients. Lancet. 1989;1(8648):1156-1160.

89. Vrieze A, et al. Metabolic effects of transplanting gut microbiota from lean donors to subjects with metabolic syndrome. Diabetologia. 2010;53:abstract 80 